\title{
Metadata and Cooperative Knowledge Management
}

\author{
Matthias Jarke ${ }^{1,2}$ and Ralf Klamma ${ }^{2}$ \\ ${ }^{1}$ Fraunhofer FIT \\ Schloß Birlinghoven, 53754 Sankt Augustin, Germany \\ ${ }^{2}$ RWTH Aachen, Informatik V \\ Ahornstr. 55, 52072 Aachen, Germany \\ jarke@cs.rwth-aachen.de
}

\begin{abstract}
Cooperative knowledge management refers to the work practice or culture facet of information systems engineering; it plays a key role especially in engineering and consulting domains. However, in comparison to technology-centered and business-process-centered meta modeling approaches (exemplified by UML and ERP), this aspect has received significantly less attention in research and is much less mature in terms of international standardization. We claim that additional interdisciplinary research effort is needed in this direction, and discuss different points of attack, largely in terms of their implications for better metadata management and meta modeling.
\end{abstract}

\section{Conceptual Modeling and Meta Modeling}

Since its invention in the mid-1970s until relatively recently, conceptual modeling was a manual documentation exercise, at best supported with some drawing tools, sometimes with syntactic correctness checks of the models, sometimes with 'automated' transformation to code frames of usually doubtful quality. Only 20 years later the efforts of standardization organizations and research groups to provide formalizations of conceptual modeling techniques and 'intelligent' tools for supporting these formalizations resulted in reasonably powerful metadata repositories which cannot only store and manipulate such models but have a reasonable formal foundation to explain why and how these models are related to each other by using meta models.

An early example has been the ConceptBase system developed in our group since the late 1980 's. ConceptBase was originally developed as a repository for lifecyclewide metadata management in information systems engineering [Jarke and Rose 1988]. Its formal basis has been a version of the Telos meta modeling language [Mylopoulos et al. 1990] which was re-axiomatized in terms of Datalog with stratified negation [Jeusfeld 1992], thus enabling reuse of all the results on query optimization, integrity checking, and incremental view maintenance developed in the logic and object database communities [Jarke et al. 1995].

On the other hand, Telos itself was an abstraction of the pioneering formalization of the widely used structured methods by [Greenspan 1984]. These methods (and this 
has not changed in their object-oriented successors such as UML) offer multiple modeling viewpoints, perspectives or contexts [Motschnig 1995]. Managing the relationships among these viewpoints has been a central design issue in ConceptBase and its applications. The key feature Telos provides for this purpose is an unlimited instantiation hierarchy with rules and constraints for defining formal semantics across multiple levels (so-called meta formulas). This allows the full range of data, metadata, meta models, meta meta models, etc. to be managed with full querying, deduction and integrity checking facilities within a single repository.

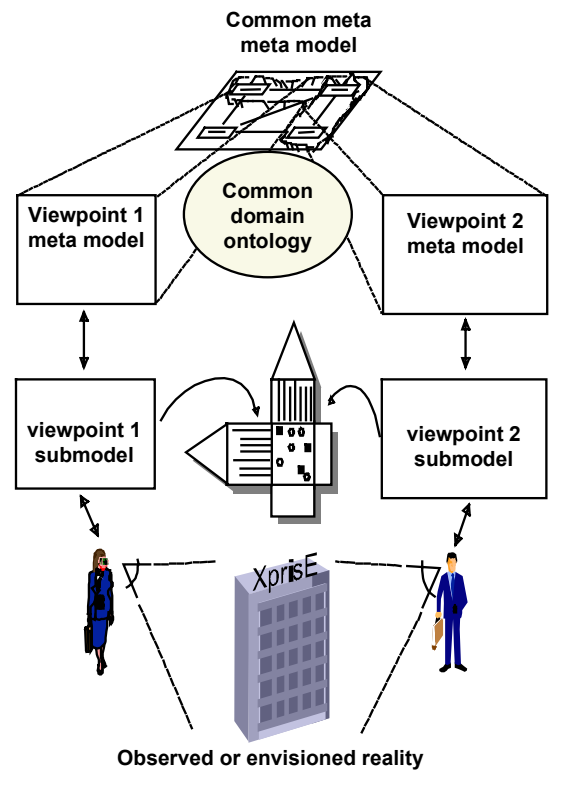

Fig. 1. Integration of heterogeneous model viewpoints via a shared meta meta model

The viewpoint resolution strategy shown in figure 1 [Nissen and Jarke 1999] focusses on the cooperative analysis of an observed or envisioned reality from multiple, interrelated viewpoints. In contrast to traditional design methods which aim at orthogonality of modeling concepts, it emphasizes judicious use of viewpoint overlaps and conflicts at all levels of instantiation for quality control and knowledge elicitation:

A shared meta meta model provides a small core ontology of the domain, similar to the ones used in engineering product and process modeling standard approaches such as STEP. The difference here is that our meta meta model comes with a fairly rich definition of meta meta concept semantics through meta-formulas which constrain the relationships of objects within and across meta models, models, or even data (the optimization of these meta-formulas efficiently constituted the key advance in the ConceptBase implementation [Jeusfeld 1992, Staudt and Jarke 2001]).

Relationships between meta models (i.e. between the constructs of different modeling formalisms used for representing heterogeneous viewpoints) was originally managed indirectly by defining each modeling construct as an instance of a specific meta meta model concept and then automatically specializing the associated meta-formulas. 

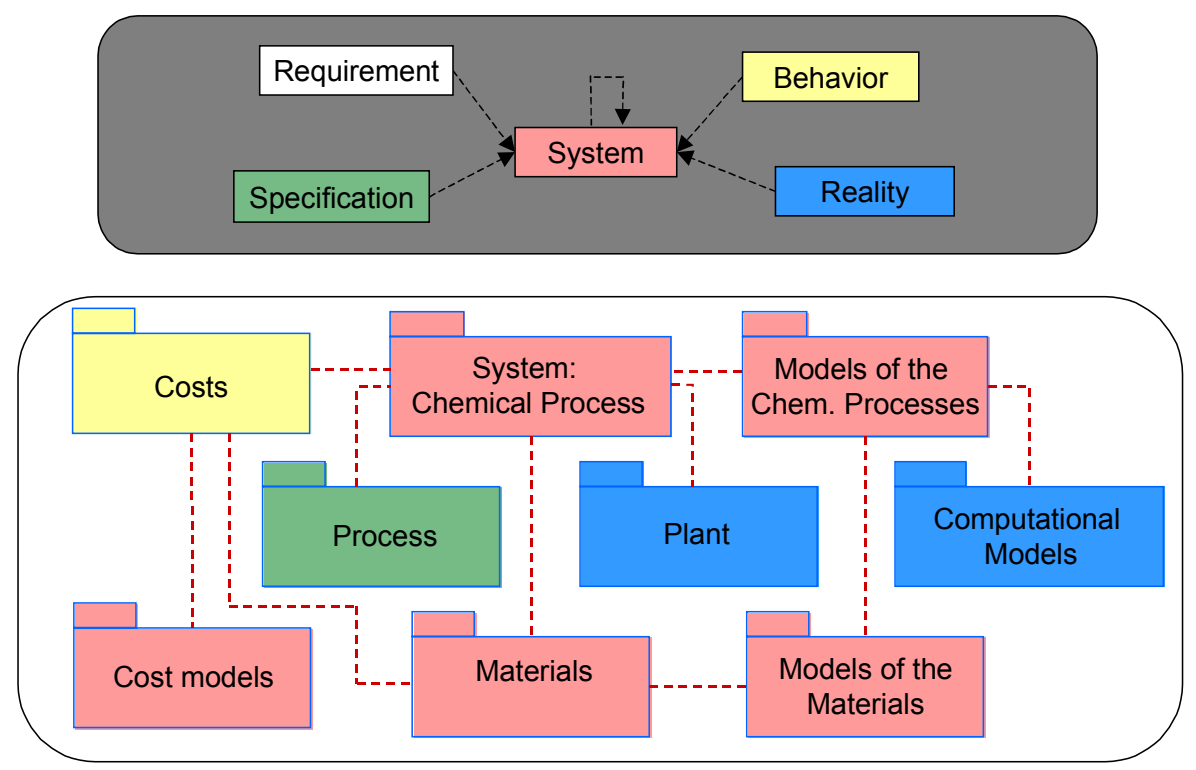

Fig. 2. Chemical engineering viewpoint meta models integrated via shared meta meta model

In complex domains with many different modeling formalisms, this leaves too many options for inter-viewpoint constraints. The definition of more elaborate domain ontologies to which the viewpoint constructs can be related is a fashionable solution [Staab et al. 2001]. In figure 2, we show a recent example from an interdisciplinary project with chemical engineers [Marquardt et al. 2001]. It illustrates the complexity of co-managing information about reality (e.g. a chemical plant and the materials it works with and produces), specified behavior (processes operating on these plants), observations of actual behavior with respect to requirements (e.g. costs), and highly complex mathematical models and simulation tools for the analysis of all the above. Under the shown system-theory inspired basic meta meta model, a rich ontology of several hundred concepts has been developed in order to facilitate modeling and cross-viewpoint analysis at a sufficiently fine-grained level. Some details about this so-called process data warehousing approach can be found in [Jarke et al. 2000].

In cases such as this one, this ontology-enriched approach can be technically supported by linking more expressive special-purpose reasoners (e.g. from description logics [Horrocks 1999]) to ConceptBase which keep the ontologies internally consistent and well-organized. On the other hand, none of these special-purpose reasoning mechanism can currently replace the initial Datalog-based optimization because they do not provide the infinite instantiation capabilities (cf. also [Calvanese et al. 2001]).

In a specific modeling process, further specialization of the inter-viewpoint analysis can be derived from the meta formulas. But again, this requires at least the identification and documentation of which model objects in the different viewpoints refer to the same phenomena in reality. Thus, as figure 1 shows, the models need not only be related through the shared meta meta model but also by a shared grounding in reality. This grounding is, in short, provided by scenario-based approaches as discussed later. 
The resulting relationships can be documented using practice-proven matrix-based representations such as the 'house of quality' from quality function deployment.

The above approach proved quite useful in applications such as business process analysis under varying theories of what constitutes good or bad business practice [Nissen and Jarke 1999], cooperation process analysis [Kethers 2002], re-engineering of both large-scale database schemas and application codes [Jeusfeld and Johnen 1995], organization of multimedia teaching materials [Dhraief et al. 2001], and the structured tracing of large-scale engineering processes [Ramesh and Jarke 2001].

While ConceptBase and a few other semi-commercial research prototypes (such as the MetaEdit which emphasizes the management of relationships between graphical notations rather than logical concepts [Kelly et al. 1996] but otherwise has a similar philosophy) created some individual early success stories, metamodeling has become mainstream only due to the rising need for metadata facilities for heterogeneous information integration, and the provisioning of relatively cheap metamodeling facilities in widespread products such as the Microsoft Meta Data Engine [Bernstein et al. 1999]. This has paved the way to information model standardization efforts in several domain research and vendor communities. But even nowadays, key questions such as a high-level algebra for manipulating large sets of complex interrelated models remain largely unanswered [Bernstein 2001]. Maybe as a consequence, even the mainstream commercial tools tend to be under-utilized with respect to their potential.

In the remainder of this paper, we focus on one particularly challenging application domain for improved metamodeling and metadata management facilities: cooperative knowledge management. In section 2, we explain the meaning of this term by contrasting it with the more established UML and ERP approaches. In section 3, we review recent theories from cultural science, organizational behavior, and engineering how successful cooperative knowledge management could operate, and discuss the implications for enhanced metadata management based on experimental solutions in a number of domains. These theories also provide some additional evidence for the importance of scenario-based approaches, not only in conceptual modeling and requirements engineering, but also in knowledge delivery and teaching. Finally, we summarize our observations and the resulting research challenges.

\section{The Culture Gap in Information Systems Engineering}

The last few years have seen major breakthroughs in the use of conceptual modeling and meta modeling in information systems engineering [Mylopoulos 1999]. The breakthrough was achieved via two different avenues.

On the one hand, starting from the successes of object-oriented programming in the late 1980's, numerous object-oriented design and analysis methodologies were proposed during the early 1990's and finally converged in the Unified Modeling Language (UML) [Rumbaugh et al. 1999]. UML basically generalizes proven programming technologies to analysis and design. Not surprisingly, the main usage of UML nowadays is in late design.

On the other hand, starting from best practice analyses in various branches of business, ERP researchers and vendors have developed standard software for the adminis- 
trative processes of enterprise resource planning (production planning and scheduling, human resources, accounting/ financials), culminating in comprehensive software packages such as SAP or Oracle Financials. These systems and models basically encode proven business practices, at the modeling level by software tools such as ARIS [Scheer 1994] or INCOME [Oberweis et al. 1994]. Coming from an organizational viewpoint, the key success factor is significantly reduced domain analysis effort, combined with the cost and risk reduction involved with using parameterized off-the-shelf software systems compared to developing software from scratch.

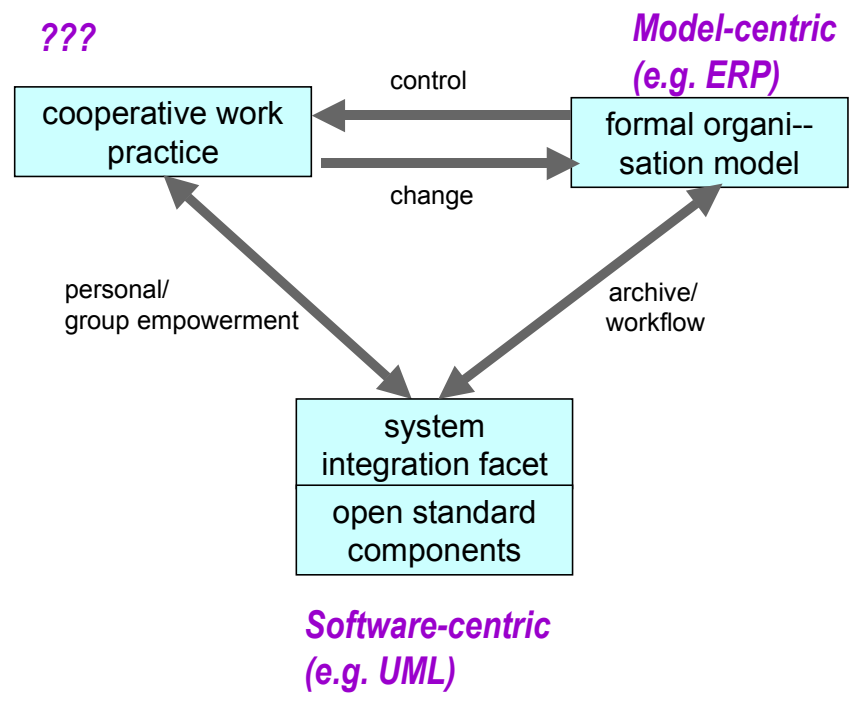

Fig. 3. The culture gap in the cooperative information systems landscape

Proponents of these two approaches see limited relevance in each others' work. For OO proponents, ERP research is some domain ontology standardization work in OMG subgroups that clearly comes in importance after having the UML itself. For ERP leaders, UML is yet another notation, 'nice-to-have' but not mission-critical; many tools focus on older notations such as ER diagrams or Petri nets. Moreover, as sketched in figure 3, neither of the approaches deeply considers work practice communities [Wenger 1998] in the core competence areas of companies. But this is exactly is where knowledge management - the creation, combination, and dissemination of knowledge within and across organizations - is most needed.

As observed by numerous researchers and practitioners [Ulich 1992] and detailed for the case of information systems engineering in [deMichelis et al. 1998], successful information systems require a flexible balance among formal organization, cooperative work practice (often also called organizational culture), and the information system technology in use. This balance is clearly missing in either of the mentioned approaches due to their lack of interaction with the organizational work practice.

The three-tier client-server architecture of ERP systems can historically be understood as bringing standardized business processes back into the once chaotic PC usage landscape [Laudon 1986]. The difficulties SAP faced to integrate the cooperation- 
oriented customer-relationship management (CRM) into their process-oriented ERP software [Plattner 1999] illustrates the point. This is despite the fact that this kind of external service-oriented collaboration (cf. [Schäl 1996]) is much more structured than internal project-oriented collaboration and knowledge management e.g. in engineering departments. Engineering, not accidentally, happen to be the one area where most businesses have not managed to implant their ERP solutions well.

Similarly, UML offers only one (albeit innovative and important) feature related to work practice: use cases of systems interaction. However, considerations of work practice are crucial not just in the requirements phase but throughout the whole systems lifecycle including actual usage. Methods for systematic use-case oriented testing, selling, user documentation, and operations management are still immature.

Within the work practice support community (e.g. CSCW), many solutions have been tried out with varying success, but no standard modeling techniques of the kind described above have emerged. Partially, this may be due to the negative attitude that many $\mathrm{CSCW}$ researchers have towards modeling itself, especially towards process modeling and workflows which they consider too restrictive. Instead, somewhat reminiscent of the database field, many approaches within this community focus on offering information and communication technology as a medium for cooperation. The usage of such systems evolves by successful experiences in a grass-root manner -provided the learning effort is low, there are practical win-win situations at each step of adoption, and the formal organization does not prevent it.

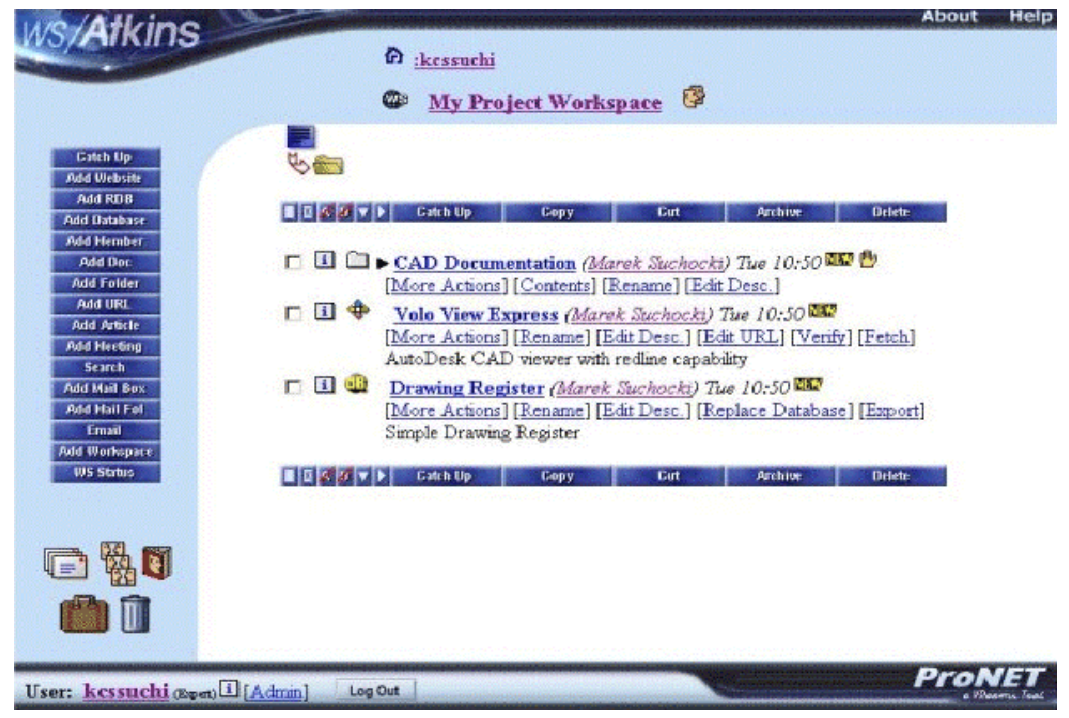

Fig. 4. The BSCW shared workspace environment

As a prototypical example, consider the grass-root diffusion of the Basic Support for Cooperative Work (BSCW) system developed at Fraunhofer FIT [Appelt 1999] in a large British civil engineering firm. The BSCW (cf. the screendump in figure 4) provides an internet-based open workspace environment organized in a folder hierarchy of multimedia documents. Users can invite other users to share their workspaces 
with certain access rights and a defined level of version management, can define how to get notified or made visually aware of changes others have made in documents, etc.

In the civil engineering firm, this system was initially adopted by a small group of engineers to manage the documented knowledge created during their participation in a European project. Recognizing and advertising the usefulness, system usage rapidly spread into many internal engineering projects throughout the company. In a next step, the system began to be used by engineering groups in joint efforts with the company's customers. Only after departments already offered this collaboration service to customers as an Application Service Provider (ASP), the formal organization finally took notice of this work practice innovation and placed it under information management within the company! Clearly, knowledge management should be able to deal more proactively with such cooperative work practice innovations.

\section{Perspectives on Cooperative Knowledge Management}

The creation and management of knowledge in heterogeneous communities of practice [Wenger 98] has fascinated researchers at least since Socrates. Of course, depending on the disciplinary viewpoint, the understanding of the involved processes and tools varies widely, even when the focus is on information systems support.

Among the many proposals, we review three theories we have found useful for our own knowledge management research. Briefly, a theory from the cultural sciences discusses how changing media influence the way knowledge is represented, distributed and evolved in cultural discourses. This motivates metadata research related to information condensation, personalization, and community awareness.

A theory from organizational behavior describes the processes how knowledge is extracted from a context of practice, manipulated for deeper understanding and creativity, and brought back to work practice. This motivates research in information escalation chains as well as in contextualized information capture and provisioning.

Finally, a theory from engineering statistics focuses on the crucial issue of refining knowledge from failures and missed opportunities. This highlights the critical role of scenario management as a complement to basic use cases, but also the need for traceability, goal analysis and cause-effect analysis in cooperative knowledge management.

\subsection{Cultural Science: Knowledge Management as Media-Enabled Discourse}

Since 1998, the German National Science Foundation (DFG) has been funding a major research center in Cologne to study the interplay between media and cultural communications in an interdisciplinary setting (www.uni-koeln.de/inter-fak/fk-427/). The theoretical basis of this effort [Jäger 2001] interprets knowledge creation and management as a cultural discourse in which the inter-medial transcription of concepts and the addressing of the created media objects within the culture play a central role. The basic idea is illustrated in figure 5 . 


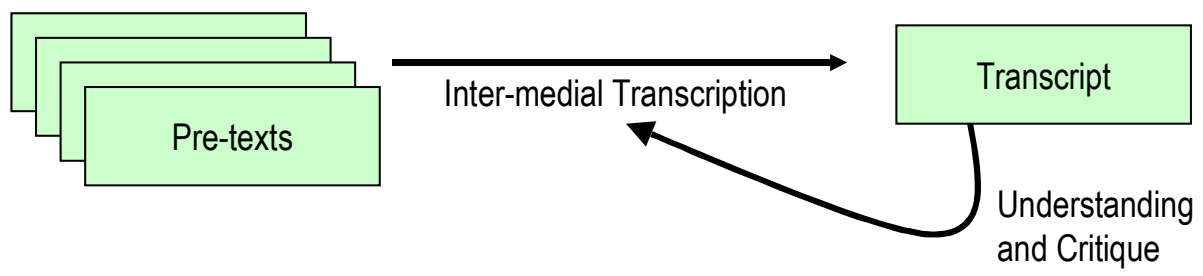

Fig. 5. Transcription creates interpretations of media in media

The key point is simple: it is impossible to develop and debate concepts separately from the media through which they are communicated. Empirical studies [Daft and Lengel 1986, Grote and Klamma 2000] show that even the mental maps of people strongly depend on the media through which they communicate effectively. This appears to argue strongly against the possibility of purely abstract conceptual modeling or ontology building, without explicit consideration how these models/ontologies are captured and communicated via media. Similar claims have incidentally been made concerning the advantages of scenario-based analysis techniques over pure conceptual modeling (for an overview cf. [Jarke et al. 1998]).

New concepts are, according to [Jäger 2001], developed by making selections from an initially unstructured set of media-based 'pre-texts' and converting them through a process called intra- or inter-medial transcription into a so-called transcript, a media object describing the new concept.

Transcription and targeted dissemination have a number of consequences:

1. It condenses and structures the set of pre-texts in terms of designate some of them as evidences, counter-examples, or points of critique, while de-emphasizing others.

2. It thus enables a new reading of the pre-texts where the kind of readership is determined by the media through which the transcript is prepared and communicated. Well-designed transcripts can significantly increase the community of practice for a certain piece of knowledge, or may intentionally focus on specific 'insiders'.

3. Thus, transcription does not only change the status of passive understanding by others but it also enables further cooperative knowledge creation by stimulating debate about the selected pre-texts and about the transcription itself.

As a prototypical example [Jäger 2001], consider a historical narrative which sheds provocative new light on the almost infinite set of historical records, thus causing a debate including criticisms of both the chosen sources and the chosen transcription.

As an engineering knowledge management example of critiquing a transcript through another one, consider a large set of natural language use cases arranged in organized in BSCW folders according to their supposed relevance to architecture components. Now we run a text mining system over the same document collection which automatically clusters the use cases according to their similarity and visually compares these clusters with the folder organization. In a multi-country software standardization project in the chemical industries, this approach served to detect errors in the placement of use cases or the relevance of use cases to multiple architectural com- 
ponents [Braunschweig et al. 1999]. However, this success was strongly correlated with the fairly standardized terminology use within chemical engineering (pointing to the relevance of empirically grounded rather than artificially constructed ontologies); a similar attempt to optimize the archive structure of newspaper articles analysis failed miserably -- journalists are trained to use inventive, non-repetitive language!

From this theoretical perspective, meta modeling offers the unique ability to custom-tailor media, thus allowing community-specific or even person-specific [Riecken 2000] transcription and addressing. Language plays a special role because it is best suited for intra-medial transcriptions, i.e. meta-discourses about itself. Thus, despite the fact that such meta-discourses tend to become overly abstract and detached from practice, metadata in computer-tractable language can play a key role to drive the tailoring of transcription and addressing media; of course, extensible instantiation hierarchies such as offered by Telos, or reflexive object-oriented formalisms can be particularly useful in this context

Transcriptions in constructed media can radically change the knowledge community. As an (admittedly not very commercial) example, a Judaistic hypothesis of how knowledge was encoded within Talmudic tractates over its development history of several centuries, has been the basis for a transcription of the traditional Mishna and Gemara rolls into a structured and annotated multi-lingual hypertext using XMLoriented metadata management [Hollender et al. 2001]. This transcript, for example, supports switching between languages such as English, German, and Hebrew while maintaining the knowledge structure through e.g. color highlighting and annotations. Such features make these texts -formerly readable only by a few rabbinic specialists accessible to beginning students and other interested parties with limited knowledge in both Hebrew and Judaistic concepts. This re-addressing has rapidly created a worldwide teaching and learning community (of course including heated debates about the adequacy of the underlying theory itself).

At a broader albeit simpler level, the full transcription and re-targeting process is supported by information brokering frameworks such as the Broker's Lounge developed in Fraunhofer FIT ([Jarke et al. 2001], see figure 6). The Broker's Lounge supports, in an interoperable way, a range of possible brokering processes - each involving particular ways of collecting and selecting pre-texts (sources, typically extacted from document management systems or the Internet), their analysis with respect to a given transcript expressed through categorization (metamodel) and conceptualization (ontology), and their selective dissemination and personalization.

However, only in much more limited and well-managed settings such as data warehousing, current transcription mechanisms involve rich semantic or media transformations, powerful quality checks, and the like. Case studies show that, in these latter cases, even nowadays organizations accept the need to manage extremely complex multi-perspective metadata at conceptual, logical, and physical levels to achieve organization-critical data quality needs [Schäfer et al. 2000].

\subsection{Business View: Knowledge as Information in a Context of Action}

While cultural scientists may see knowledge creation and management as a value in itself, knowledge for business has value only in the context of organizational action 
[Mandl and Gerstenmaier 2000]. Probably the best-known theory along these lines was proposed by [Nonaka and Takeuchi 1995]. As the mapping of their process model into our framework in figure 7 shows, Nonaka and Takeuchi see knowledge creation and management mostly as an exercise of transferring knowledge from one context of work practice to another context of work practice.

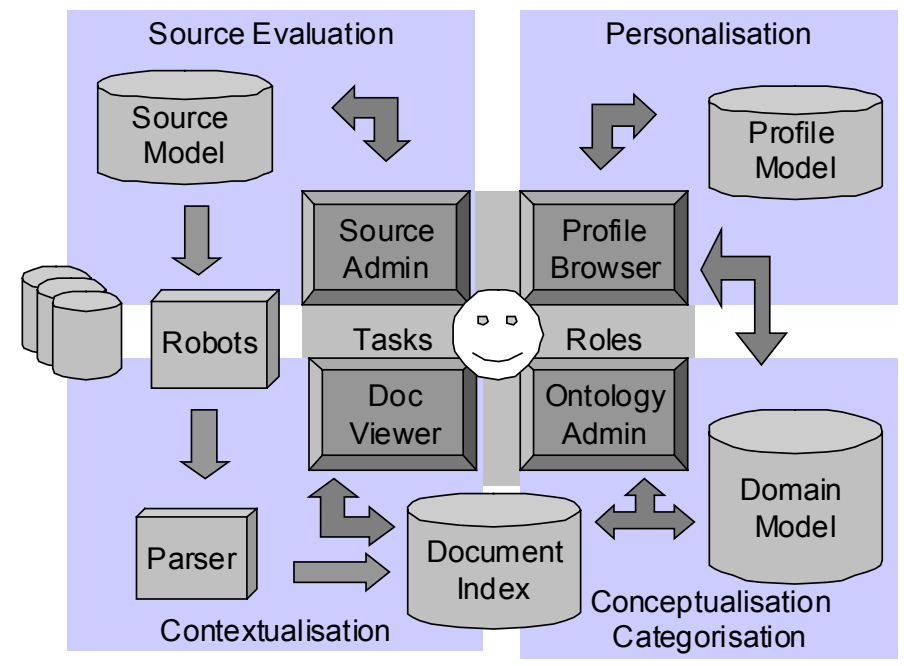

Fig. 6. The Broker's Lounge environment for adaptable information brokering

This transfer can either be handled implicitly by being aware of what other people in the culture do; this is called socialization. It is interesting to note that the CSCW community has, in the last few years, placed great emphasis on supporting awareness in geographically and temporally distributed work settings to enable this kind of transfer [Gross and Specht 2001]. Relevant metadata include interest and attention models, privacy models, models of space, time, and task settings, all with a goal to make awareness as informative and unobtrusive as possible in comparison to the same-place same-time setting. Such metadata can, for example, be employed in some kind of event server which resolves which events in a distributed setting should be captured and how to broker them to interested parties [Prinz 1999].

Alternatively, knowledge transfer can go through an explicit de-contextualization and memorization step (called externalization), followed by formal manipulation of the resulting (media) artifacts (called combination) and by the re-contextualization into the same or an alternative work practice context (called internalization). Most computer science work in knowledge management, including approaches from casebased reasoning (drawing analogies from past practice cases to new practice cases), from data/text mining (finding general patterns in large data or case sets), and even the media-enabled transcription approach discussed above relates to the conversion and connection tasks related to the combination step [O'Leary 1998]. In contrast, broad information systems support for the full cycle in figure 3 remains a major challenge. 


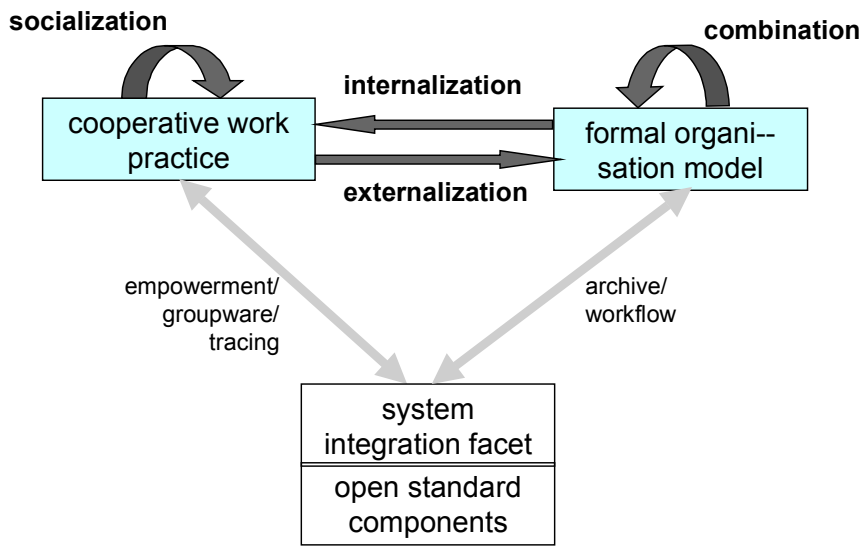

Fig. 7. Nonaka's process model of organizational knowledge creation adapted to our framework

\subsection{Engineering View: Knowledge is Defined by What Is Different or Wrong}

Another critical success factor is how knowledge management can actually stimulate a community of practice to engage in this process, i.e. what actually drives the knowledge management cycle. An answer found in many engineering-oriented organizations and communities is: recognized failures and missed opportunities. A typical quotation from a senior engineering manager in the automotive industry: 'we judge our engineers by how well they deviate from the textbook'.

Starting from statistical test theory, [Dhar 1998] has argued that organizations recognize failures of attempted action (type I errors) relatively easily and therefore have a natural tendency towards increasing caution and bureaucracy. However, it is equally important not to miss opportunities (avoid type II errors), e.g. by creating autonomy for (real or simulated) experiments as well as enabling their sharing.

The sources for such innovative ideas can be everywhere in work practice, including the usage practice of customers. Helpdesk systems are becoming an important way to achieve this. The traditional way of looking at these systems is that they form subcommunities organized in escalation chains such that, if the knowledge of the first-line helpdesk people is insufficient to answer a call, it can be escalated to second-line service specialists, and - in the worst case - back all the way into design-level product quality circles (cf. figure 8). The driving force of all this (and in some sense the source of the knowledge!) is the customer who complains or asks for help.

In such escalation chains, knowledge creation happens in two directions. The obvious one, realized in many products on the market, is a gradual improvement of service quality by transferring knowledge from designers to the helpdesk personnel.

The less obvious one - where most organizations still have severe problems - is the mining of the complaint and solution experiences for improving design and servicability within and across product families. Besides the combination techniques required for representing and generalizing such experiences, the transcription of this knowledge for use in the design communities and the targeting to the places where it is most rele- 
vant for organizational improvement prove to be critical [Klamma and Jarke 1998]. Metadata include escalation workflows, competence networks, and organizational principles of how the content of the organizational memory is structured and linked to situated process models along the lines discussed, e.g. in [Rolland 1998].

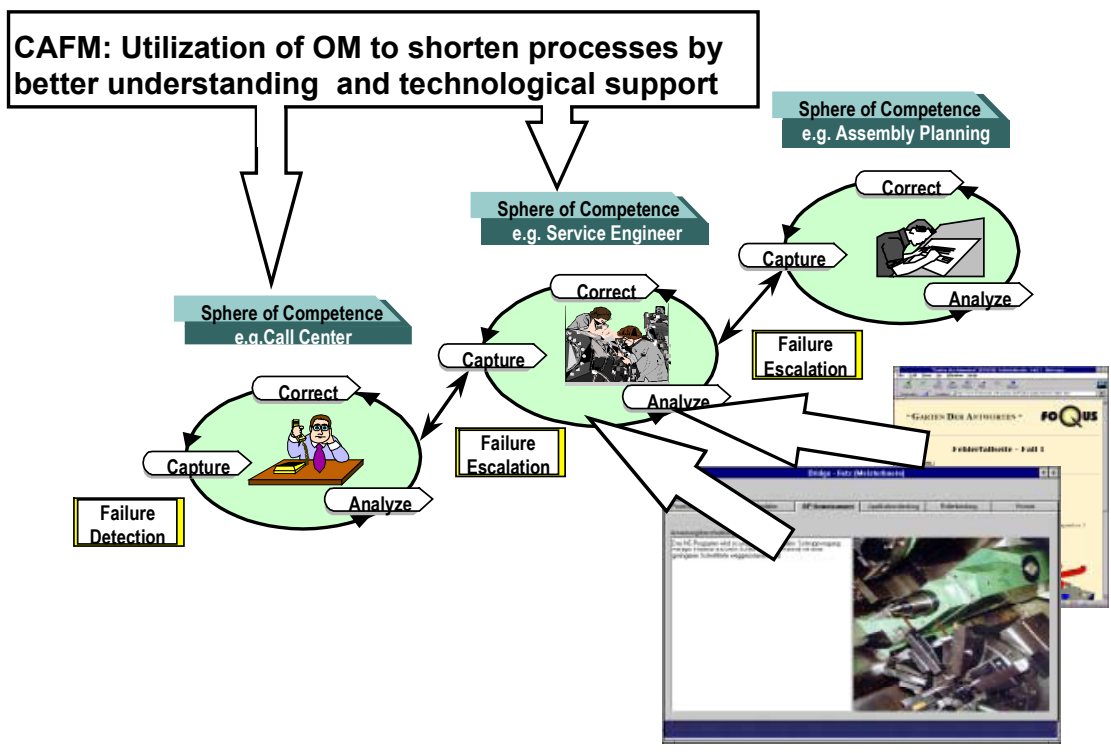

Fig. 8. Failure-driven knowledge creation: escalation chains in complaint management

\section{Implications for Knowledge Modeling and Management}

When we link the three theoretical approaches sketched for cooperative knowledge management to the discussion of metadata in the first part of this paper, the lessons can be summarized as follows. The theories

emphasize the careful design of media in addition to underlying formalism, including attention focussing by suitable transcription techniques

emphasize the linkage of knowledge to action, with the implication of seamless decontextualization from action, re-contextualization into action, and careful design of communications networks in distributed work practice

emphasize the need to accept the full richness of reality in a knowledge management system, including the learning from product and process errors as well as from opportunities recognized by customers.

It is interesting to note that many of these arguments reflect similar discussions on scenario-based design where scenarios are seen as middle-ground abstractions for organizational memory, because (1) they delay commitment while encouraging participation, (2) they focus on use and differences, and (3) improve memorization and reuse (cf. [Jarke et al. 1998] for more details). Indeed, the CREWS framework shown in figure 9 in a certain sense already reflects the circular nature of goal discovery and 
observation focus discussed in the transcriptivity theory of [Jäger 2001], and its goal orientation reflects the need to handle exceptions systematically [Sutcliffe et al. 1998].

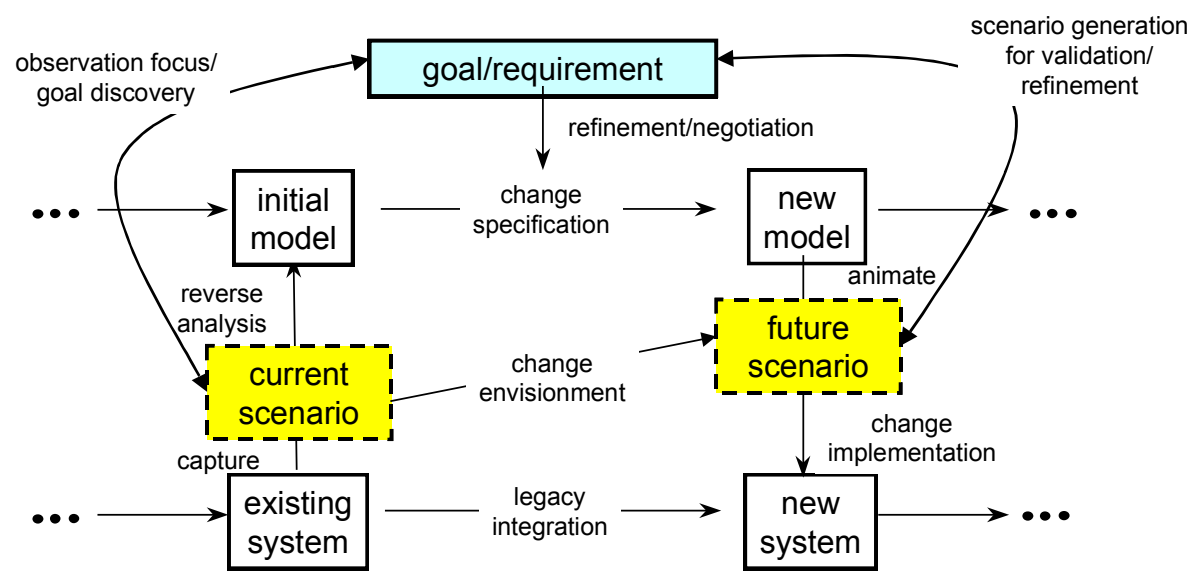

Fig. 9. Integrating models and media in scenario-based requirements engineering

However, cooperative knowledge management goes beyond requirements engineering because of its broader scope both in terms of organizational coverage and in terms of the product and process lifecycles. One interesting direction we are currently pursuing with a group at MIT [Fendt 2001] is the systematic usage of scenarios organized around meta models for knowledge dissemination via eLearning.

As a final example, figure 10 shows a prototype Virtual Entrepreneurship Lab [Klamma et al. 2001] in which collections of video clip scenarios (taken mostly from interviews with well-known international entrepreneurs, venture capitalists, and the like) are arranged according to a conceptual meta model derived from a suitable underlying theory which, however, is never formally shown to the students. Only the base categories of the domain meta meta model are shown to the students as dimensions of interest (cf. vertically arranged buttons on the right of figure 10). Selecting one or more such dimensions creates a thumbnail gallery of scenarios arranged around a larger viewing window to which the student can drag and play a video of interest. Based on this choice, the video gallery can then re-arrange itself according to the meta models; but the student can also create his or her own view in the lower left part of the figure. Initial tests in a high-tech entrepreneurship class at RWTH Aachen show very encouraging results compared with traditional teaching techniques.

A crucial success factor of the Virtual Entrepreneurship Lab is the availability of the MPEG-7 ISO standard for multimedia metadata [Avaro and Salembier 2001] which make this integration of media and models no longer an exotic adventure. In this manner, the requirements for advanced metadata and meta modeling identified in this paper are actually beginning to be addressed by standards which give hope that our quest for a maturity of cooperative knowledge management similar to UML or ERP approaches may actually make significant advances in the next few years. 


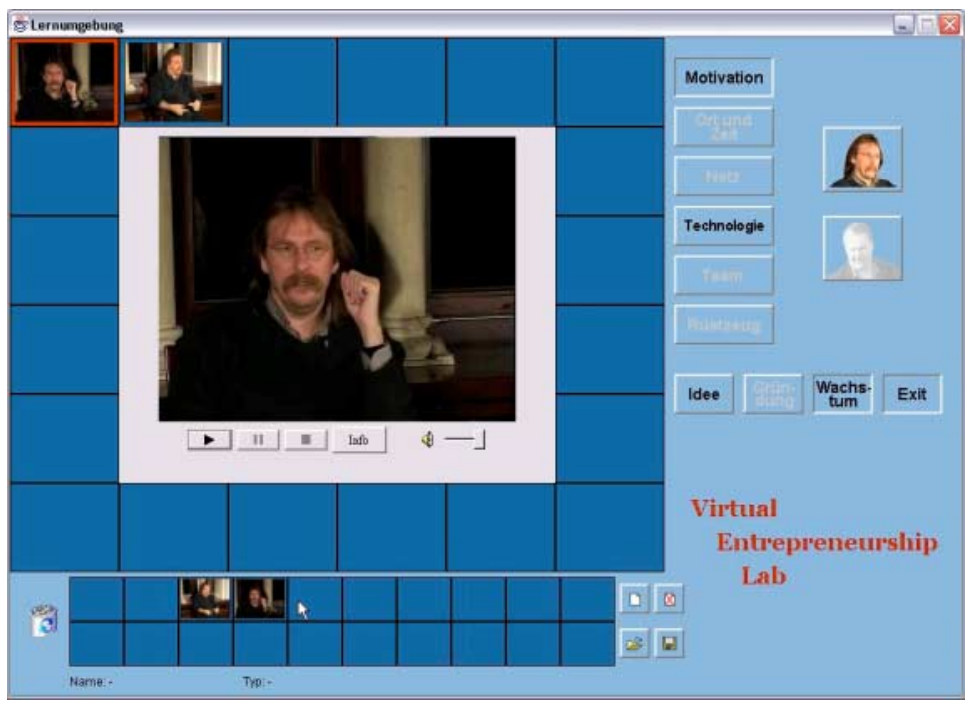

Fig. 10. Scenario-based approach to knowledge delivery: The virtual entrepreneurship lab

\section{Acknowledgements}

This work was supported by German National Science Foundation (DFG) within the collaborative research centers SFB/FK 427 "Media and cultural communication" and SFB 476 "IMPROVE“. We like to thank Roland Klemke and Wolfgang Prinz for the fruitful discussions.

\section{References}

1. Appelt, W.: WWW Based Collaboration with the BSCW System. Proc. SOFSEM'99, Milovy, Czech Republic, LNCS 1725 (1999), 66-78.

2. Avaro, O., Salembier, P.: MPEG-7 Systems: overview, IEEE Transactions on Circuits and Systems for Video Technology 11, 6 (2001): 760-764.

3. Bernstein, P. A.: Generic model management - a database infrastructure for schema management. Proc. CoopIS 01, Trento, Italy, LNCS 2172, Springer 2001, 1-6.

4. Bernstein, P. A., Bergstraesser, T., Carlson, J., Pal, S., Sanders, P., Shutt, D.: Microsoft repository version 2 and the open information model. Inform. Systems 24, 2 (1999):71-98.

5. Braunschweig, B., Jarke, M., Becks, A., Köller, J., Tresp, C.: Designing standards for open simulation environments: a computer-supported use case approach. Proc. $9^{\text {th }}$ Intl. Symposium on Systems Engineering (INCOSE 99), Brighton, UK, vol. 2, 69-76.

6. Calvanese, D., De Giacomo, G., Lenzerini, M., Nardi, D., Rosati, R.: Data integration in data warehousing. Intl. J. Cooperative Information Systems, spring 2001. 
7. Daft, R. L., Lengel, R. H.: Organizational Information Requirements, Media Richness and Structural Design, Management Science, 32, 5 (1986): 554-571.

8. Dhar,V. Data mining in finance: using counterfactuals to generate knowledge from organizational information systems. Information Systems 23, 7 (1998): 423437

9. Dhraief, H., Nejdl, W., Wolf, B., Wolpers, M.: Open Learning Repositories and Metadata Modeling, Proc. 1st Semantic Web Working Symposium, Stanford 2001, 495-514.

10. Fendt, K.: Contextualizing content. In Knecht, M., v. Hammerstein, K. (eds.): Languages across the curriculum. National East Asian Language Ctr., Columbus, Oh 2001, 201-223.

11. Ghezzi, C., Nuseibeh, B., eds. Special Section on Managing Inconsistency in Software Development. IEEE Trans. Software Engineering 24, 11 (1998), and 25, 6 (1999).

12. Greenspan, S.: Requirements modeling - a knowledge representation approach to software requirements definition. Ph.D. Thesis, Computer Science Dept., Toronto 1994.

13. Grote, K., Klamma, R.: Media and Semantic Relations. Comparison of Individual and Organizational Knowledge Structures. Dagstuhl Seminar "Knowledge Management. An Interdisciplinary Approach", Schloss Dagstuhl, Germany, 09.07.2000 - 14.07.2000.

14. Gross, T., Specht, M. Awareness in context-aware information systems. Proc. Mensch \& Computer 01, Bonn 2001.

15. Hollender, E., Klamma, R., Börner-Klein, D., Jarke, M. A comprehensive study environment for a Talmudic tractate., Dutch Studies by Foundation for Near Eastern Languages and Literatures (DS-NELL) (in print).

16. Horrocks, I.: FaCT and iFaCT. Proc. International Workshop on Description Logics (DL'99), Linköping, Sweden, CEUR-WS 22, 133-135.

17. Jarke, M., Bui, X. T., Carroll, J. M. Scenario management: an interdisciplinary approach. Requirements Eng. 3, 3-4 (1998): 155-173.

18. Jarke, M., R. Gallersdörfer, M. A. Jeusfeld, M. Staudt and S. Eherer (1995). ConceptBase - a deductive object base for meta data management. Intell. Inform. S. 4, 2 (1995): 167-192.

19. Jarke, M., Klemke, R., Nick, A. Broker's Lounge - an environment for multidimensional user-adaptive knowledge management. Proc. HICSS 34, Wailea, Hw, 2001 .

20. Jarke, M., List, T., Köller, J.: The challenge of process data warehousing. Proc. $26^{\text {th }}$ Intl. Conf. Very Large Databases, Cairo 2000, 473-483.

21. Jarke, M., Rose, T.: Managing the evolution of information systems. Proc. SIGMOD 1988, Chicago, IL.

22. Jeusfeld, M.: Änderungskontrolle in deduktiven Objektbanken. DISKI 19, infix Publ. Bad Honnef, Germany, 1992 (Diss. Univ. Passau 1992).

23. Jeusfeld, M., Johnen, U.: An executable meta model for re-engineering database schemas, Intl. J. Cooperative Information Systems 4, 2-3 (1995): 237-258. 
24. Kelly, S., Lyytinen, K., Rossi, M.: MetaEdit+ - a fully configurable multi-user and multi-tool CASE and CAME environment. Proc. CAiSE 96, Heraklion, Greece, 1996, 1-21.

25. Kethers, S.: Capturing, formalising, and analysing cooperation processes - a case study. Proc. ECIS 2002, Gdansk, Poland (to appear).

26. Klamma, R., Jarke, M. Driving the organizational learning cycle: the case of computer-aided failure management. Proc. 6th ECIS Conf. (Aix-en-Provence 1998), 378-392.

27. Klamma, R., Moog, P., Wulf, V.: How to start a company? The Virtual Entrepreneurship Lab (VEL) as a teaching aid and training instrument for potential startups (in German). Proc. G-Forum Jahreskonferenz, Lüneburg/Germany 2001.

28. Laudon, K.: From PCs to managerial workstations: organizational environment and management policy in the financial industry. In Jarke, M. (ed.): Managers, Micros, and Mainframes - Integrating Systems for End Users. John Wiley \& Sons 1986.

29. Mandl, H., Gerstenmaier, J. (ed.): Die Kluft zwischen Wissen und Handeln. Hogrefe 2000.

30. Marquardt, W.; von Wedel, L.; Bayer, B.: Perspectives on lifecycle process modeling. Proc. Foundations of Computer-Aided Process Design, AIChE Symposium Series 323, Vol. 96 (2000), 192-214.

31. deMichelis, G., Dubois, E., Jarke, M., Matthes, F., Mylopoulos, J., Papazoglou, M., Schmidt, J.W., Woo, C., Yu, E. A three-faceted view of information systems: the challenge of change. Comm. ACM 41, 12 (1998): 64-70.

32. Motschnig-Pitrik, R. An integrating view on the viewing abstraction - contexts and perspectives in software development. Journal of Systems Integration 5, 1 (1995): 23-60.

33. Mylopoulos, J. Information modeling in the time of the revolution. Information Systems 23, 3-4 (1998): 127-156.

34. Mylopoulos, J., Borgida, A., Jarke, M., Koubarakis, M.: Telos - a language for managing knowledge about information systems. ACM Trans. Inform. Systems 8, 4 (1990): 327-362.

35. Nissen, H. W., Jarke, M. Repository support for multi-perspective requirements engineering. Information Systems 24, 2 (1999), 131-158.

36. Nonaka, I., Takeuchi, H. The Knowledge-Creating Company, Oxford Univ. Press, 1995.

37. Oberweis, A., Scherrer, G., Stucky, W.: INCOME/STAR - methodology and tools for the development of distributed information systems. Inform. Systems 19, 8 (1994):643-660.

38. O'Leary, D. Knowledge management systems: converting and connecting. IEEE Intelligent Systems 1998(5):30-33.

39. Papazoglou, M. P., Schlageter, G. (eds.). Cooperative Information Systems: Trends and Directions. Academic Press, 1998.

40. Plattner, H.: Customer relationship management. In Scheer, A.-W., Nüttgens, M. (eds.): Electronic Business Engineering Proc. WI 99, Saarbrücken), Physica 1999, 1-12. 
41. Prinz, W. NESSIE: An Awareness Environment for Cooperative Settings. Proc. ECSCW'99: 6th European Conference on Computer Supported Cooperative Work, Copenhagen 1999, Kluwer Academic Publishers, 391-410.

42. Ramesh, B., Jarke, M.: Towards reference models for requirements traceability. IEEE Trans. Software Eng. 27,1 (2001): 58-93.

43. Riecken, D. (Hrsg.): Special Issue on Personalization. Comm. ACM 43, 8 (2000).

44. Rolland, C.: A comprehensive view of process engineering. Proc. CAiSE 98 (Pisa), 1-24.

45. Rumbaugh, J., Jacobson, I., Booch, G. The Unified Modeling Language Reference Manual. Addison-Wesley 1999.

46. Schäfer, E., Becker, J.-D., Jarke, M. DB-Prism - integrated data warehouses and knowledge networks for bank controlling. Proc. 26th VLDB Conf., Cairo, Egypt, 715-718.

47. Schäl, Th. Workflow management systems for process organizations. Springer 1996.

48. Scheer, A.-W. Business Process Engineering. Springer 1994.

49. Staab, S. Schnurr, H.-P., Studer, R., Sure, Y.: Knowledge processes and ontologies. IEEE Intelligent Systems 16, 1 (2001).

50. Staudt, M., Jarke, M.: View management support in advanced knowledge base servers. Intelligent Information Systems 15, 3 (2000): 253-285.

51. Sutcliffe, A., Maiden. N., Minocha, S., Manuel, D.:Supporting scenario-based requirements engineering. IEEE Trans. Software Eng. 24, 12 (1998).

52. Ulich, E. Arbeitspsychologie. Stuttgart: Schäffer-Pöschel 1992.

53. Wenger, E.: Communities of Practice - Learning, Meaning, and Identiy, Cambridge University Press, Cambridge, UK, 1998. 